\title{
Cervicoplastia ampliada para correção de alterações cervicais
}

\author{
Expanded cervicoplasty to correction of cervical changes
}

\author{
Daniel Nunes ${ }^{1}$ \\ JOÃO ILGENFRITZ JR. ${ }^{2}$ \\ Giovani Pires Viana ${ }^{1}$ \\ Giovani ANdré Pires Viana ${ }^{1}$ \\ Kleder Gomes De Almeida ${ }^{1}$ \\ Marcelo Cury ${ }^{2}$ \\ Elaine LeaL ${ }^{2}$ \\ Diogo Gomes Augusto 3
}

Trabalho realizado na clínica privada do autor principal, Campo Grande, MS, Brasil.

Trabalho apresentado para ascensão à categoria de membro titular da SBCP.

Artigo submetido pelo SGP (Sistema de Gestão de Publicações) da RBCP.

Artigo recebido: 24/11/2010 Artigo aceito: 15/3/2011

\begin{abstract}
RESUMO
Introdução: A cervicoplastia é considerada parte importante na cirurgia do rejuvenescimento facial. Apesar das diversas técnicas já descritas, a busca pelo tratamento ideal ainda persiste. As dificuldades aumentam nos casos considerados complexos, em que a flacidez excessiva de pele e a ptose muscular pronunciada prejudicam a manutenção dos resultados a longo prazo. Objetivos: No intuito de discutir as opções técnicas disponíveis para a cervicoplastia e apresentar a rotina cirúrgica utilizada pelo autor - com ênfase no tratamento ampliado sobre o músculo platisma - é que este estudo foi delineado. Método: Dezoito pacientes foram submetidos ao round lifting associado à confecção de retalhos musculares de platisma, na linha médio-cervical, e à retirada parcial da gordura subplatismal. Os pacientes foram acompanhados por 12 meses e 0 resultado pós-operatório tardio foi avaliado por cinco cirurgiões plásticos da SBCP. Resultados: Quatro pacientes foram excluídos do estudo por não retornarem para o acompanhamento pósoperatório tardio. Dos 14 pacientes avaliados, $7(50 \%)$ tiveram o resultado classificado como excelente, $5(35,7 \%)$ como moderado e $2(14,3 \%)$ como fraco. Nenhum paciente teve o resultado classificado como perfeito ou ruim. Conclusão: A cervicoplastia ampliada proporcionou a obtenção de resultados satisfatórios, mesmo em casos difíceis. Acreditamos que a técnica é uma alternativa válida para o cirurgião plástico, podendo contribuir para que obtenhamos um resultado pós-operatório agradável, natural e mantido com o tempo.
\end{abstract}

Descritores: Ritidoplastia. Cervicoplastia. Músculos do Pescoço/cirurgia.

\begin{abstract}
Introduction: Cervicoplasty is considered an important part of facial surgery. Despite the various techniques already described, the search for optimal treatment still persists, especially in complex or difficult cases, where the skin excess and the muscular ptosis affect the maintenance of long-term results. Objective: This study was designed to discuss the technical options available to cervicoplasty and present the routine used by the author - with emphasis on platisma muscle extended treatment. Method: Eighteen patients were submitted to "Round Lifting" associated with a platisma muscle flap in cervical mid-line and partial removal of subplatismal fat. The patients were followed for 12 months and late postoperative outcome was evaluated by five of SBCP plastic surgeons. Results: Four patients were excluded for not returning for follow-up. Of the 14 patients evaluated, 7 (50\%) patients had an excellent result, $5(35.7 \%)$ patients moderate and $2(14.3 \%)$ weak. No patient had the result classified as perfect or poor. Conclusion: The expanded cervicoplasty described here provided great results, even in difficult cases. We believe that the technique is a valid alternative to the plastic surgeon, which can help you obtain a pleasing postoperative result, natural and maintained over time.
\end{abstract}

Keywords: Rhytidoplasty. Cervicoplasty. Neck Muscles/surgery.

\footnotetext{
1. Cirurgião Plástico, Membro titular da Sociedade Brasileira de Cirurgia Plástica.

2. Cirurgião Plástico, Membro especialista da Sociedade Brasileira de Cirurgia Plástica.

3. Médico estagiário do Serviço.
} 


\section{INTRODUÇÃO}

Desde sua origem, a cervicoplastia é considerada um elemento importante na cirurgia do rejuvenescimento facial, sendo que o sucesso do procedimento continua vinculado à obtenção de um pescoço harmônico e natural ${ }^{1,2}$.

Apesar das diversas técnicas já descritas, um "padrãoouro" não foi estabelecido. A busca pelo tratamento ideal ainda persiste, especialmente nos casos considerados mais complexos ou difíceis, em que a flacidez excessiva de pele e a ptose muscular pronunciada prejudicam a manutenção dos resultados a longo prazo. Ainda hoje, o tratamento do "pescoço difícil" - termo celebrizado por McKinney ${ }^{3}$ (Quadro 1) - permanece sendo considerado um verdadeiro desafio para o cirurgião plástico ${ }^{4-10}$.

A cervicoplastia moderna é a reunião de fundamentos propostos por diversos autores, como Millard et al. ${ }^{11}$, que, em 1968, sistematizaram o acesso ao pescoço através do sulco submentoniano; por Baker et al. ${ }^{12}$, que, em 1973, demonstraram a importância da aproximação dos bordos mediais do músculo platisma e da fixação de suas margens laterais no músculo esternocleidomastoideo; e por Feldeman ${ }^{13}$, que, em 1990, defendeu a necessidade de uma plicatura vigorosa no músculo platisma na linha média.

Posteriormente, autores como McKinney ${ }^{3}$, Patrocínio \& Patricínio $^{6}$, Pontes \& Pontes $^{14}$, entre outros, defenderam a necessidade de uma abordagem mais agressiva na cervicoplastia de casos complexos, incluindo a ressecção da gordura subplatismal e a confecção de retalhos musculares na linha médio-cervical ${ }^{8,15-17}$.

Desta forma, no intuito de discutir as opções técnicas disponíveis para o tratamento da região cervical nas ritidoplastias e apresentar a rotina cirúrgica utilizada pelo autor - com ênfase no tratamento ampliado sobre o músculo platisma - é que este estudo foi delineado.

\section{MÉTODO}

Foram avaliados, prospectivamente, todos os pacientes submetidos à cervicoplastia na clínica privada do autor

\begin{tabular}{|c|c|}
\hline \multicolumn{2}{|c|}{ Quadro 1 - Classificação de McKinney ${ }^{3}$. } \\
\hline Grau & Região Cervical \\
\hline I & $\begin{array}{c}\text { Bandas platismais poucos } \\
\text { visíveis }\end{array}$ \\
\hline II & $\begin{array}{l}\text { Bandas platismais } \\
\text { moderadamente visíveis }\end{array}$ \\
\hline III & $\begin{array}{c}\text { Bandas platismais muito } \\
\text { visíveis }\end{array}$ \\
\hline IV & $\begin{array}{c}\text { Bandas platismais muito } \\
\text { visíveis e flacidez excessiva } \\
\text { de pele }\end{array}$ \\
\hline
\end{tabular}

principal do estudo, no período de janeiro de 2007 a junho de 2009.

Para o tratamento do terço inferior da face, foi utilizada a técnica clássica do round lifting, preconizada por Pitanguy ${ }^{18}$, associada à confecção de retalhos musculares do platisma na linha médio-cervical e com a retirada parcial da gordura subplatismal. Segue o sumário da técnica cirúrgica utilizada na cervicoplastia.

\section{Descrição da Cervicoplastia}

O procedimento é realizado sob leve sedação, administrada pelo anestesiologista, com fentanila $(0,05 \mathrm{mg} / \mathrm{ml}$ $\mathrm{EV})$ e midazolan $(5 \mathrm{mg} / \mathrm{ml} \mathrm{EV})$. O paciente é posicionado em decúbito dorsal, com flexão posterior da cabeça e a antissepsia é realizada com solução degermante de clorexidina a $2 \%$, seguida da colocação de campos estéreis. O cirurgião posiciona-se atrás da cabeça do paciente e, com azul de metileno, realiza a marcação da incisão no sulco submentoneano, que varia de 4-6 cm. Em seguida, inicia-se a infiltração anestésica da região cervical com cerca de $100 \mathrm{ml}$ de uma solução contendo lidocaína $0,125 \%$ com adrenalina 1:200.000. Com bisturi lâmina 11, realiza-se uma pequena incisão, sobre a marcação prévia, e inicia-se então uma lipoaspiração da região médio-cervical, que, dependendo das características do caso, pode ser leve ou moderada. Em seguida, com bisturi lâmina 15 , incisa-se no sulco submentoneano marcado e disseca-se amplamente a região com tesoura de Metzembaum. A dissecção tem como limites anatômicos a linha inferior da mandíbula, a borda anterior do músculo esternocleidomastoideo e a cartilagem cricoide, evidenciando-se amplamente o músculo platisma. Ressecase, então, a porção média de cada músculo platisma, quando estes se apresentam em excesso. Em seguida, a borda medial restante de cada músculo platisma é dissecada lateralmente por cerca de $2,5 \mathrm{~cm}$, sendo liberada da musculatura subjacente, confeccionando-se dois retalhos musculares. Realizase, então, a retirada da parcial da gordura subplatismal com tesoura de Metzembaum. Em seguida, aproximam-se os retalhos platismais, na linha média, com sutura simples com mononylon 3.0, em camada única, com cerca de $5-8 \mathrm{~cm}$ de comprimento, da incisão submentoneana até a região do osso hioide (Figura 1).

Procede-se, então, à sequência clássica do round lifting ${ }^{18}$, destacando-se a importância das plicaturas realizadas no sistema SMAS-Platisma - na região parótido-malar e infraauricular (junto à borda lateral do músculo esternocleidomastoideo) - para a potencialização dos resultados da cervicoplastia (Figura 2).

Com o término da cirurgia, após a realização dos demais procedimentos ancilares indicados para cada caso, realiza-se a colocação de dreno aspirativo, a sutura da pele e a aplicação de curativo compressivo. Após as primeiras 24 horas 


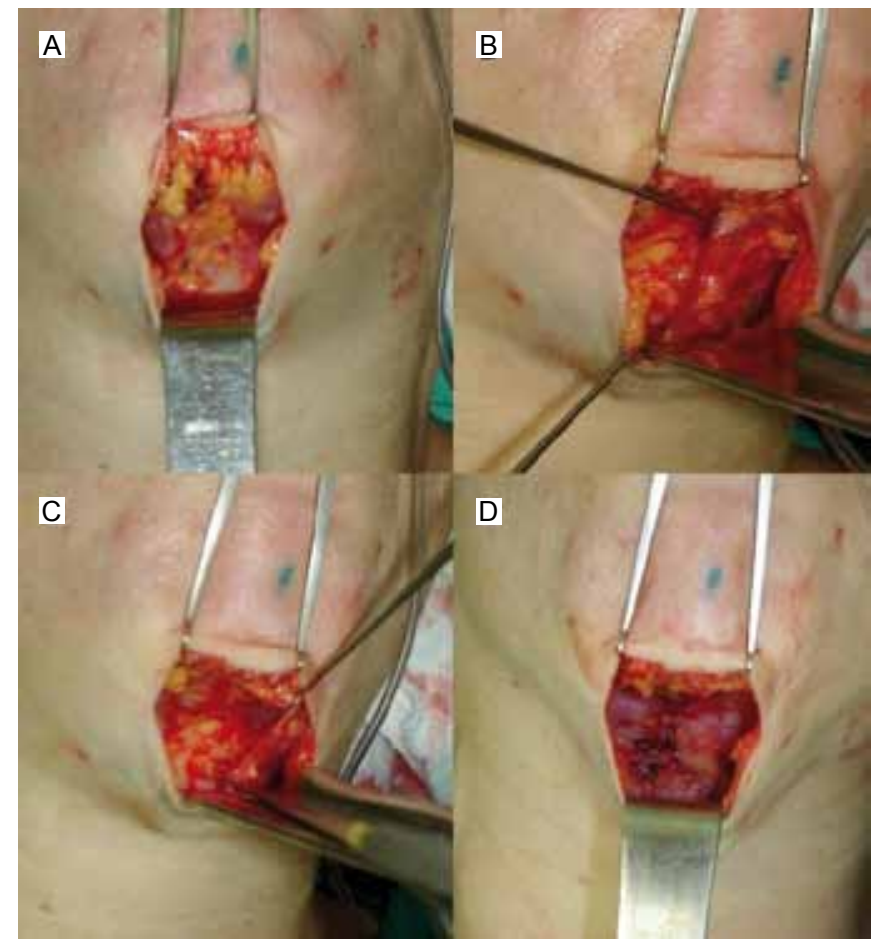

Figura 1 -Aspectos do transoperatório da paciente K. A: Amplo descolamento cervical e visualização das bordas mediais dos músculos platismas; B: Descolamento da borda medial do músculo platisma esquerdo com visualização da gordura subplatismal; $\boldsymbol{C}$ : Elevação do retalho platismal esquerdo com retirada parcial da gordura subplatismal, D: Avançamento e sutura dos dois retalhos musculares na linha médio-cervical. de internação, o dreno é retirado e o curativo substituído por malha de compressão cervical específica. O paciente recebe alta hospitalar e é acompanhado ambulatorialmente. Os pontos são retirados a partir do $7^{\circ}$ pós-operatório. Aos pacientes é recomendado o repouso absoluto por 7 dias e a drenagem linfática, realizada com fisioterapeuta especializado, é iniciada no $3^{\circ}$ dia de pós-operatório.

Após a cirurgia, os pacientes foram acompanhados, semanalmente, no primeiro mês e, bimestralmente, até o sétimo mês. Doze meses após o procedimento, foi realizada a última avaliação. Os pacientes que não retornaram para todas as revisões foram excluídos do estudo.

As fotos do pré-operatório e do pós-operatório tardio (12 meses) foram avaliadas por cinco cirurgiões plásticos da Sociedade Brasileira de Cirurgia Plástica (SBCP) - JI Jr, MC, EL, GAPV, GV. A cervicoplastia foi avaliada segundo método modificado de Antell \& Orsesk ${ }^{10}$, sendo: 1 ponto $=$ sem melhora ou resultado fraco; 2 pontos $=$ melhora leve; 3 pontos $=$ melhora moderada; 4 pontos $=$ melhora importante; e 5 pontos $=$ resultado considerado perfeito.

As avaliações de cada paciente foram somadas e o resultado final classificou a cirurgia em: ruim (5-8 pontos); fraco (9-13 pontos); moderado (14-18 pontos); excelente (19-23 pontos); e perfeito (24-25 pontos).

Em caráter ilustrativo e didático, os autores apresenta imagens de dissecções anatômicas realizadas em peças formolizadas, que demonstram o plano de descolamento do músculo platisma, a retirada da gordura subplatismal e a confecção dos retalhos musculares na linha média (Figura 3).

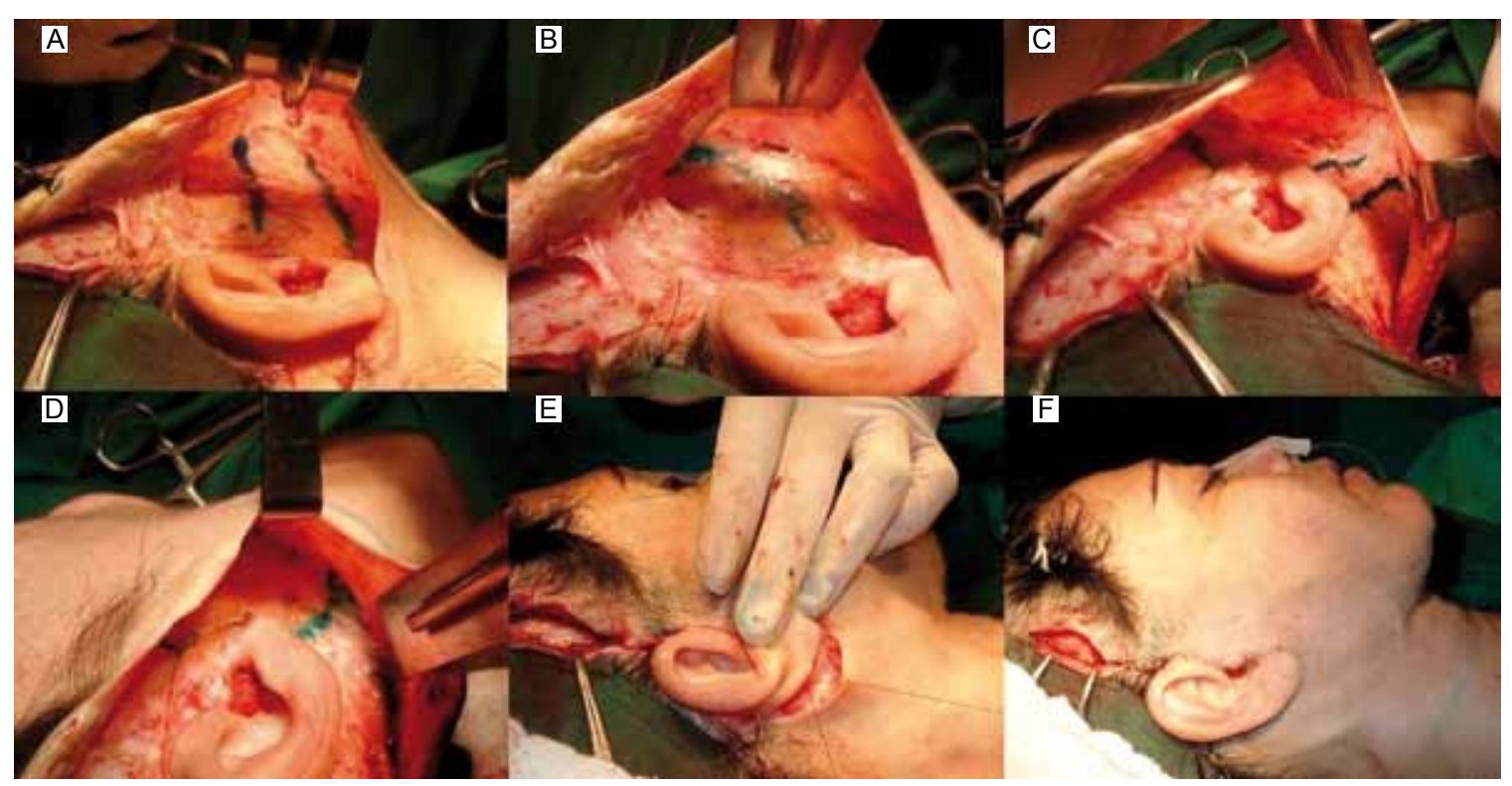

Figura 2 - Aspectos do transoperatório da paciente K. A: Marcação da área de plicatura na região parótido-malar direita; B: Resultado da plicatura; $\boldsymbol{C}:$ Marcação da área de plicatura na região infra-auricular direita; D: Resultado da plicatura; E: Confecção de sutura profunda de sustentação do retalho cervical na concha auricular direita; F: Aspecto final da cervicoplastia. 


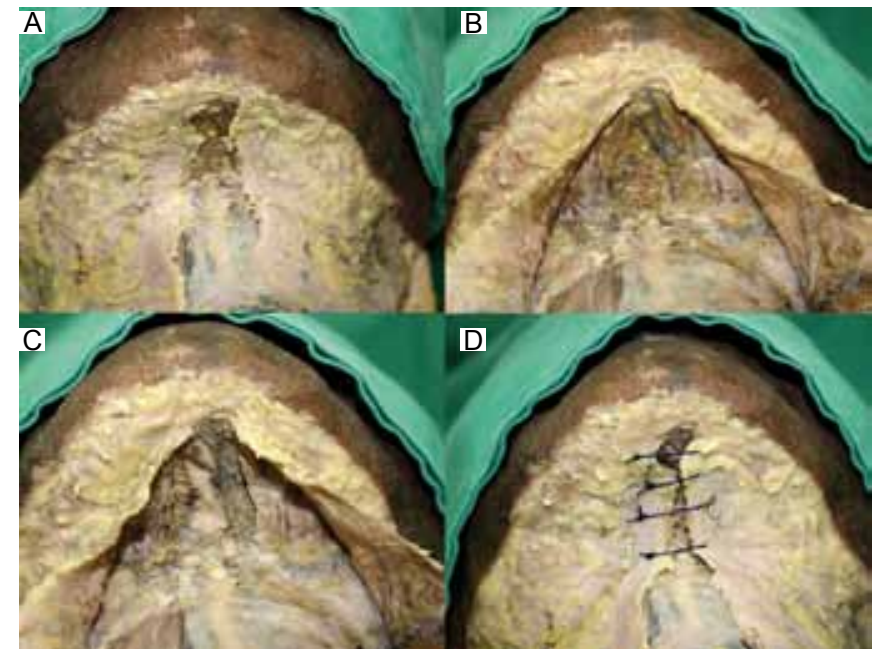

Figura 3 - Aspectos da dissecção de região cervical de cadáver. A: Retirada da cobertura de pele e tecido celular subcutâneo, com exposição dos músculos platismais; B: Dissecção e elevação muscular com exposição da gordura subplatismal; $\boldsymbol{C}$ : Retirada da gordura subplatismal, com manutenção do plexo vascular e exposição da musculatura subjacente; D: Aproximação ilustrativa dos retalhos platismais, sem secções na musculatura.

\section{RESULTADOS}

No período do estudo, 18 pacientes foram submetidos à cervicoplastia ampliada. Quatro foram excluídos por não retornarem às consultas de pós-operatório de acompanhamento tardio. As características da amostra são apresentadas na Tabela 1.

\begin{tabular}{c|c|c|c|c}
\hline \multicolumn{5}{c}{ Tabela 1 - Características dos pacientes em estudo. } \\
\hline Paciente & Idade & Cor & Sexo & McKinney $^{3}$ \\
\hline A & 59 & B & F & IV \\
\hline B & 44 & B & F & III \\
\hline C & 59 & B & M & IV \\
\hline D & 51 & B & F & IV \\
\hline E & 54 & B & F & IV \\
\hline F & 52 & B & F & IV \\
\hline G & 50 & B & F & III \\
\hline H & 65 & B & F & IV \\
\hline I & 48 & B & F & III \\
\hline J & 55 & B & F & IV \\
\hline K & 51 & B & F & IV \\
\hline L & 49 & B & F & III \\
\hline M & 58 & B & M & IV \\
\hline N & 67 & B & F & IV \\
\hline
\end{tabular}

O procedimento cirúrgico teve duração média de $225 \pm$ 47 minutos. Não foi encontrada dificuldade na confecção do retalho muscular de platisma, bem como na ressecção da gordura subplatismal. Todos os pacientes receberam alta com cerca de 24 horas de internação. A recuperação pósoperatória foi considerada satisfatória, sendo que apenas uma paciente (paciente $\mathrm{D}$, fumante e McKinney IV) evoluiu com hematoma e necrose superficial em região infra-auricular bilateralmente, resultando em cicatriz local hipocrômica. Os demais pacientes evoluíram sem intercorrências.

O resultado da avaliação da cervicoplastia, com as notas de cada cirurgião plástico, é apresentado na Tabela 2. A Figura 4 apresenta a comparação entre a classificação de McKinney e a nota final de cada paciente.

No $12^{\circ}$ mês de pós-operatório, 7 (50\%) pacientes tiveram o resultado classificado como excelente, $5(35,7 \%)$, como moderado, e $2(14,3 \%)$, como fraco. Nenhum paciente teve o resultado classificado como perfeito ou como ruim.

Nas Figuras 5 a 10, são apresentados alguns dos pacientes operados (fotos de pré e pós-operatório de 12 meses), visão frontal, perfil direito e esquerdo).

\section{DISCUSSÃo}

Parece consenso na literatura que muito ainda está por ser discutido sobre a abordagem do "pescoço difícil". A necessidade do desenvolvimento de opções técnicas mais efetivas, no intuito de oferecer resultados superiores, é referida por diversos autores há anos $3,6,8,13,16,19,20$.

A cervicoplastia ampliada, que associa diversas condutas cirúrgicas, é uma alternativa muito indicada por cirurgiões plásticos que almejam um tratamento cervical de qualidade em casos complexos ${ }^{9,15}$. Segundo Cardoso de Castro et al. ${ }^{2,15}$, nestes casos, procedimentos menores - mais indicados por cirurgiões inexperientes - além de não estarem livres de complicações, fornecem resultados limitados e efêmeros.

A partir disso, buscando a excelência de resultados para casos difíceis, a cervicoplastia ampliada aqui descrita associou diversas opções técnicas: a lipoaspiração da região cervical ${ }^{21}$; 0 descolamento amplo do tecido celular-subcutâneo do pescoço; a retirada parcial da gordura subplatismal ${ }^{6}$; a confecção e o avançamento de retalhos musculares de platisma na linha média ${ }^{22}$; o tratamento do complexo SMAS-Platisma através de plicaturas na região malar (vetor cranial) e na região do músculo esternocleidomastoideo (vetor lateral) ${ }^{15,18}$.

Atualmente, a lipoaspiração superficial da região cervical é utilizada pela maioria dos autores. Ela garante a abordagem homogênea do pescoço, diminuindo a ocorrência de irregularidades e facilitando o descolamento posterior com tesoura ${ }^{2,8,16,17,21,23}$.

$\mathrm{O}$ descolamento subcutâneo amplo da região cervical, através da incisão submentoneana, também é considerado de fundamental importância na cervicoplastia de casos 


\begin{tabular}{c|c|c|c|c|c|c|c}
\hline \multicolumn{7}{c}{ Tabela 2 - Apresentação das notas de cada avaliador e a classificação do resultado da cervicoplastia. } \\
\hline Paciente & JI Jr & MC & EL & GA & GV & $\begin{array}{c}\text { Soma das } \\
\text { Notas }\end{array}$ & $\begin{array}{c}\text { Classificação do } \\
\text { Resultado }\end{array}$ \\
\hline A & 5 & 4 & 5 & 4 & 4 & 21 & Excelente \\
\hline B & 4 & 3 & 3 & 3 & 2 & 15 & Moderado \\
\hline C & 4 & 3 & 4 & 2 & 3 & 16 & Moderado \\
\hline D & 3 & 3 & 2 & 1 & 2 & 11 & Fraco \\
\hline E & 4 & 4 & 4 & 3 & 2 & 17 & Moderado \\
\hline F & 3 & 4 & 4 & 2 & 1 & 14 & Moderado \\
\hline G & 4 & 5 & 5 & 4 & 4 & 22 & Excelente \\
\hline H & 4 & 4 & 4 & 4 & 4 & 20 & Excelente \\
\hline I & 4 & 5 & 5 & 5 & 4 & 23 & Excelente \\
\hline J & 5 & 4 & 4 & 5 & 5 & 23 & Excelente \\
\hline K & 5 & 4 & 5 & 4 & 4 & 22 & Excelente \\
\hline L & 4 & 4 & 5 & 2 & 2 & 17 & Moderado \\
\hline M & 3 & 4 & 3 & 2 & 1 & 13 & Fraco \\
\hline N & 4 & 4 & 4 & 4 & 4 & 20 & Excelente \\
\hline
\end{tabular}

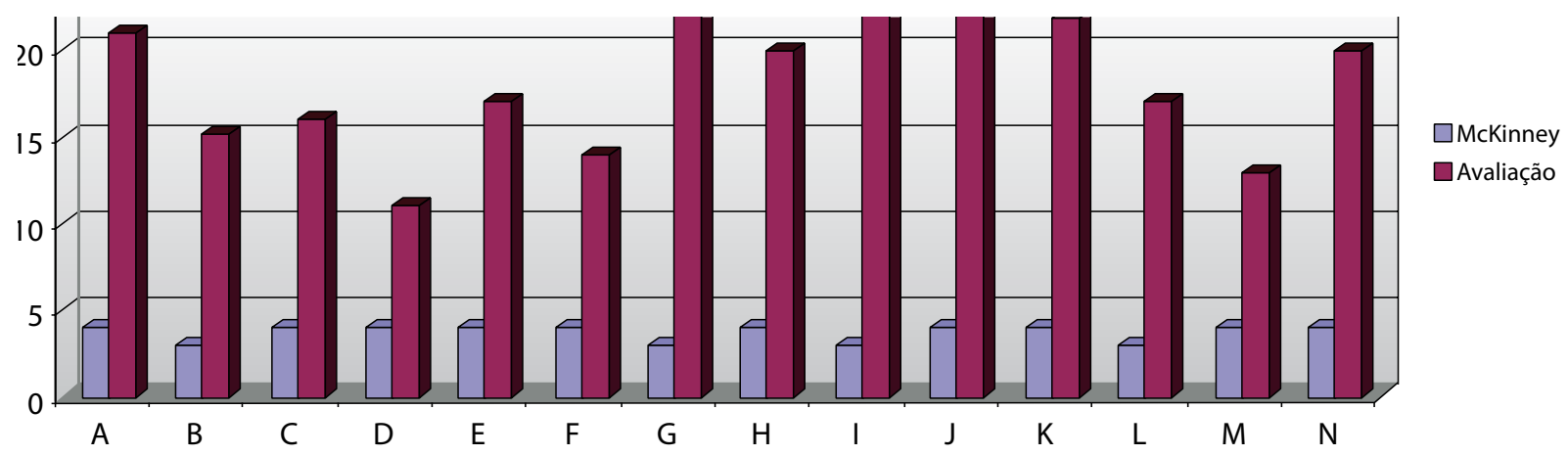

Figura 4 - Relação entre a classificação de McKinney e avaliação dos pacientes.

difíceis ${ }^{14,15,24}$. Pontes $\&$ Pontes ${ }^{14}$, Marchac et al. ${ }^{23}$, Cardoso de Castro $^{25}$ e Gomes Filho et al. ${ }^{24}$ defendem sua utilização, por facilitar a abordagem das estruturas do pescoço e a posterior acomodação da pele. Alguns autores, todavia, defendem descolamentos menores ${ }^{16,21}$. Concordamos que dissecções limitadas preservam a qualidade da pele e minimizam as chances de complicações, quando comparadas às ritidoplastias extensas $^{16,21}$, no entanto, acreditamos, assim como Cardoso de Castro et al. ${ }^{15}$, que maiores descolamentos favorecem o melhor tratamento do pescoço, principalmente em casos complexos.

Dentre as técnicas aqui utilizadas, a ressecção da gordura subplatismal é a menos referida na literatura. Pontes \& Pontes ${ }^{14}$

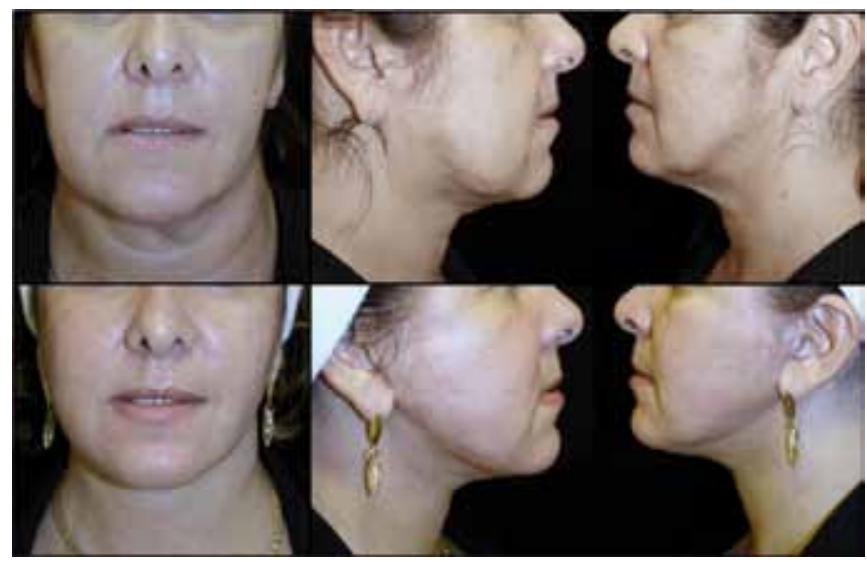

Figura 5 - Fotos do pré e do pós-operatório da paciente $G$. 


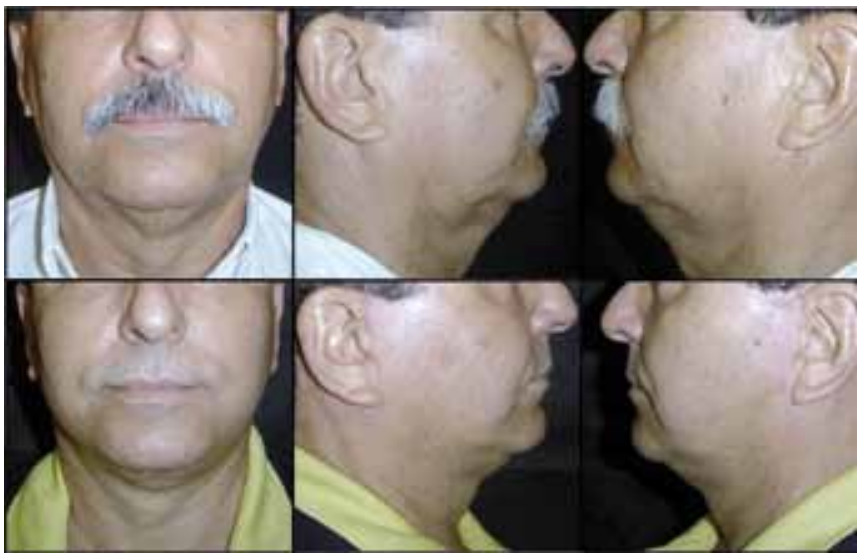

Figura 6 - Fotos do pré e do pós-operatório do paciente M.

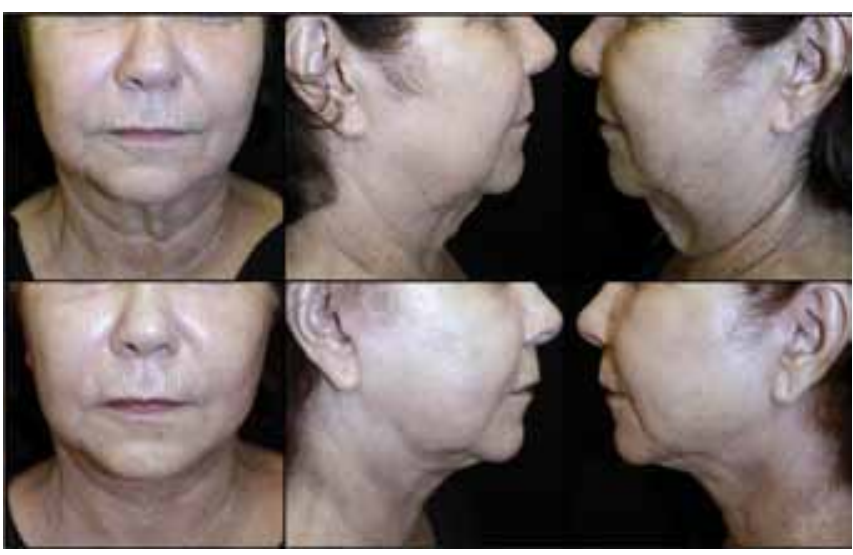

Figura 7 - Fotos do pré e do pós-operatório da paciente $H$.

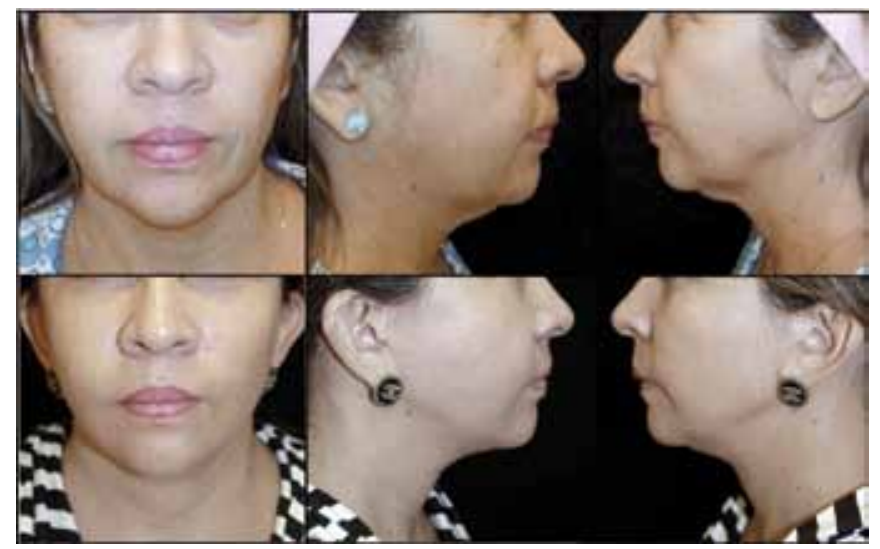

Figura 8 -Fotos do pré e do pós-operatório da paciente J.

defendem sua retirada, alegando que, com ela, os resultados pós-operatórios são superiores. Segundo Patrocínio \& Patrocínio $^{6}$, a retirada parcial da gordura subplatismal permite

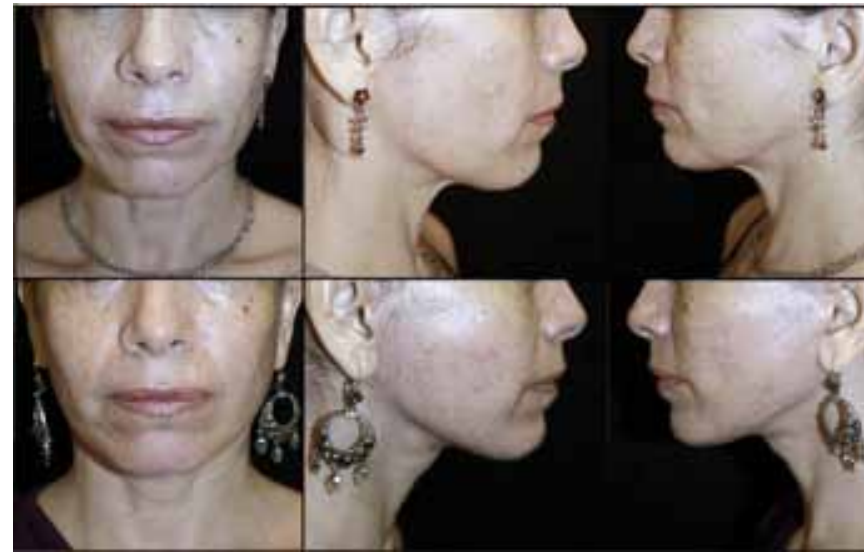

Figura 9 - Fotos do pré e do pós-operatório da paciente $K$.

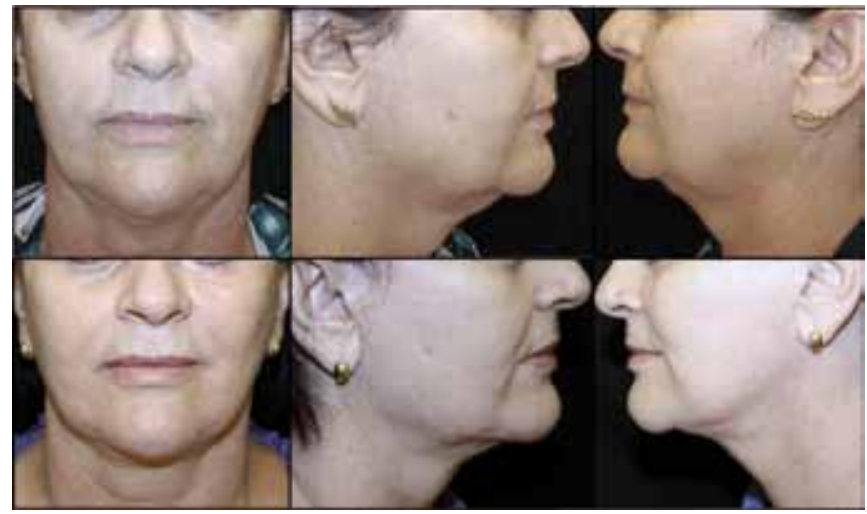

Figura 10 - Fotos do pré e do pós-operatório da paciente E.

maior avanço do músculo platisma em direção à linha média, diminuindo a tensão sobre o mesmo e valorizando o resultado da cirurgia cervical ${ }^{6}$. Zins \& Fardo $^{16}$, Knize $^{8}$, Gryskiewicz $^{17} \mathrm{e}$ Cardoso de Castro $^{2}$ defendem a ressecção da gordura subplatismal quando esta está em excesso. Neves et al. ${ }^{26}$, no entanto, discordam dos autores anteriores, referindo que a retirada da gordura subplatismal oferece mais riscos que benefícios. Cardoso de Castro ${ }^{2}$ alerta que, se a gordura for ressecada em demasia, pode-se determinar a formação de inconvenientes irregularidades na pele do submento. Segundo Zins \& Fardo ${ }^{16}$, grandes defensores da técnica, a ressecção da gordura subplatismal não deve ultrapassar a borda inferior do músculo digástrico. A partir dos resultados aqui apresentados, acreditamos que o tratamento da gordura subplatismal permite a melhor migração das bordas mediais dos retalhos do platisma, minimizando a tensão na sutura da linha média, permitindo resultados mais duradouros e naturais, acrescentando mínima morbidade à cervicoplastia.

A confecção dos retalhos musculares de platisma, apesar de descrita há anos, também é pouco valorizada na literatura 
atualmente. De modo geral, no que concerne ao tratamento do músculo platisma na linha média, a maioria dos autores defende apenas a plicatura muscular simples ${ }^{8,16}$. Tal procedimento reposiciona bem os elementos de sustentação do pescoço, oferecendo, no entanto, resultados efêmeros e desapontadores em casos mais complexos - com flacidez tecidual acentuada ${ }^{2,5,6,9,15}$. Desde de Connell ${ }^{22}$, em 1978, diversos autores vêm referindo excelentes resultados com os retalhos musculares de platisma na linha média do pescoço. Aston $^{27}$, em 1979, celebrizou a importância da confecção dos retalhos musculares, criando o que foi posteriormente chamado de "plastimoplastia de Aston". Zins \& Fardo ${ }^{16}$ apresentaram excelentes resultados com a técnica. As dissecções anatômicas aqui apresentadas demonstram a existência de um plano de clivagem bem definido entre a borda medial do músculo platisma e a musculatura subjacente. Tal plano, já havia sido identificado na borda lateral do músculo platisma nas dissecções realizadas por Labbé et al. ${ }^{9}$. Durante a realização das cervicoplastias, a confecção dos retalhos musculares de platisma (direito e esquerdo) foi facilitada pela realização da dissecção muscular ampla no plano referido. A retirada da gordura subplatismal também facilitou a migração dos retalhos musculares, realizada sem tensão, evitando a necessidade das secções do músculo platisma. Aston ${ }^{27}$, Zins \& Fardo $^{16}$ e Cardoso de Castro $^{2}$ preconizam a secção dos bordos mediais do músculo platisma, na região inferior ao osso hioide. Aston ${ }^{27}$ enfatiza que, sem estas secções, a confecção dos retalhos musculares esqueletiza o pescoço. Neves et al. ${ }^{26}$ referiram, ainda, que, sem as secções de Aston, teríamos importante dificuldade na deglutição com a técnica. Não realizamos as secções musculares no platisma, pois acreditamos que, com a associação técnicas aqui descritas, alcançamos resultados satisfatórios com menor morbidade, além de evitarmos o enfraquecimento da musculatura platismal ${ }^{8}$.

Concordamos com os autores que valorizam o tratamento do complexo SMAS-Platisma como parte fundamental da cervicoplastia de casos difíceis - associada à abordagem médio-cervical já descrita ${ }^{2,9,15,25}$. A plicatura horizontal do SMAS na região malar garante que o vetor da ascensão do terço médio seja vertical e não somente látero-superior. Tal ascensão corrige a flacidez na região média da mandíbula ${ }^{15}$. A plicatura vertical infra-auricular, fixada sobre o músculo esternocleidomastoideo, garante a tração lateral do músculo platisma, considerada essencial para a obtenção de um perfil cervical harmônico ${ }^{9,23}$. Entendemos que abordagem profunda, apenas na linha média do pescoço, acaba por tratar de forma inadequada a linha da mandíbula e por determinar a ptose das glândulas submandibulares ${ }^{5,15}$.

A análise do resultado da cirurgia é fundamental para avaliarmos o sucesso ou não da técnica utilizada. Concordamos com Cardoso de Castro et al. ${ }^{15}$, que consideram ser muito difícil, subjetivo e variável o julgamento do resultado de uma cirurgia facial. Patrocínio \& Patrocínio ${ }^{6}$, por exemplo, mediram apenas o grau de satisfação das pacientes nos pósoperatório. Labbé et al. ${ }^{9}$ creditam pouco valor científico à avaliação própria dos pacientes. Ellenbogen \& $\mathrm{Karling}^{28}$, Flores et al. ${ }^{7}$, Labbé et al. ${ }^{9}$ e Gryskiewicz ${ }^{17}$ utilizaram metodologias de avaliação diversas comparando ângulos céfaloperfilométricos diferentes, o que dificulta sua aplicabilidade. Buscando opções mais simples e objetivas, que possibilitassem a comparação com outras técnicas, adotamos aqui a metodologia proposta por Antell \& Orsesk ${ }^{10}$. Utilizando a avaliação de pontos específicos no pré e no pós-operatório, os autores desenvolveram um método simples e reprodutível, que se mostrou eficaz e rápido.

O momento de avaliação do pós-operatório também é muito discutido na literatura. Ellenbogen \& $\mathrm{Karling}^{28}$, Antell \& Orsesk ${ }^{10}$, Gryskiewicz ${ }^{17}$, assim como Labbé et al. ${ }^{9}$, defendem que o tempo mínimo para avaliarmos adequadamente o resultado de uma cervicoplastia é de 12 meses. De outro modo, Cardoso de Castro et al. ${ }^{29}$ e Patrocínio \& Patrocínio $^{6}$ acreditam que o resultado pode ser avaliado em quatro a seis meses. Por utilizarmos a metodologia de Antell $\&$ Orsesk $^{10}$, nossos resultados foram avaliados 12 meses após a cervicoplastia.

Os resultados aqui apresentados demonstraram que, mesmo em casos difíceis, a cervicoplastia ampliada obteve resultados satisfatórios, duradouros e com baixo índice de complicações. Antell \& Orsesk ${ }^{10}$ compararam técnicas diversas (mais e menos agressivas), aplicadas em 8 pares de gêmeos monozigóticos, e relataram resultados semelhantes aos aqui apresentados: $44 \%$ de resultados excelentes, $25 \%$ de moderados e $31 \%$ de fracos. Entre outros aspectos, a comparação rigorosa com outros estudos e técnicas ficou prejudicada pela utilização de metodologia diversa ou pelo tipo de análise empregada (ausente em diversas publicações) (5,6,9,28. $^{\text {. }}$.

$\mathrm{Chia}^{30}$, em 2008, fez considerações sobre o tratamento do terço médio que acreditamos podem ser utilizadas para a cirurgia do terço inferior da face. Segundo o autor, especialmente na cirurgia do rejuvenescimento facial, para obtermos sucesso é necessário que combinemos os seguintes princípios: o descolamento amplo do tecido a ser elevado, a abordagem direta da estrutura a ser tratada, a atenuação das forças musculares opositoras; o reposicionamento e a fixação em estruturas firmes ou rígidas ${ }^{30}$. A cervicoplastia ampliada aqui apresentada procurou seguir estes princípios, no intuito de obtermos resultados mais duradouros.

\section{CONCLUSÃo}

O resultado de uma cervicoplastia, especialmente para pacientes com flacidez local excessiva, está intimamente relacionado à associação de técnicas utilizadas no procedimento. A partir de uma abordagem múltipla e ampliada, 
que incluiu a confecção de retalhos musculares de platisma e a retirada parcial da gordura subplatismal, obtivemos resultados satisfatórios, mesmo em casos difíceis. Acreditamos que a cervicoplastia ampliada aqui descrita constitui uma alternativa válida para o cirurgião plástico, podendo contribuir para que obtenhamos um resultado pós-operatório agradável, natural e mantido com o tempo.

\section{REFERÊNCIAS}

1. Jonhson JB, Hardley RC. The aging face. In: Converse JM, ed. Reconstructive plastic surgery. Philadelphia:WB Saunders;1964. p.1328-49.

2. Cardoso de Castro $\mathrm{C}$. The changing role of platysma in face lifting. Plast Reconstr Surg. 2000;105(2):764-75.

3. McKinney P. The management of platysma bands. Plast Reconstr Surg. 1996;98(6):999-1006.

4. Miller TA. Face-lift: which technique? Plast Reconstr Surg. 1997;100(2):501.

5. Benito J, Righesso R. Tratamento cirúrgico da região cervical associado a suturas de suspensão platismal com fios de politetrafluoroetileno. Rev Bras Cir Plást. 2007;22(4):202-8.

6. Patrocínio LG, Patrocínio JA. Cervicoplastia anterior. Rev Bras Otorrinolaringol. 2004;70(5):597-601

7. Flores D, Sperli A, Freitas JOG, Lino AP. Avanços no estudo perfilométrico aplicados ao rejuvenescimento facial. Rev Soc Bras Cir Plást. 2000;15(3):67-78.

8. Knize DM. Limited incision submental lipectomy and platysmaplasty. Plast Reconstr Surg. 1998;101(2):473-81.

9. Labbé D, Franco RG, Nicolas J. Platysma suspension and platysmaplasty during neck lift: anatomical study and analysis of 30 cases. Plast Reconstr Surg. 2006;117(6):2001-7.

10. Antell DE, Orseck MJ. A comparison of face lift techniques in eight consecutive sets of identical twins. Plast Reconstr Surg. 2007;120(6):1667-73.

11. Millard DR, Pigott RW, Hedo A. Submandibular lipectomy. Plast Reconstr Surg. 1968;41(6):513-22.

12. Baker TJ, Gordon HL, Whitlow DR. Our present technique for rhytidectomy. Plast Reconstr Surg. 1973;52(3):232-6.

13. Feldman JJ. Corset platysmaplasty. Plast Reconstr Surg. 1990;85(3):333-43.
14. Pontes R, Pontes G. Ritidoplastia no homem. In: Mélega JM, Baroudi $\mathrm{R}$, eds. Cirurgia plástica fundamentos e arte: cirurgia estética. Rio de Janeiro:Medsi;2003. p.87-98.

15. Cardoso de Castro C, Aboudib Jr. JHC, Carvalho S. Região cervical: tecido adiposo, SMAS, platisma. In: Mélega JM, Baroudi R, eds. Cirurgia plástica fundamentos e arte: cirurgia estética. Rio de Janeiro:Medsi;2003. p.43-57.

16. Zins JE, Fardo D. The "anterior-only" approach to neck rejuvenation: an alternative to face lift surgery. Plast Reconstr Surg. 2005;115(6):1761-8.

17. Gryskiewicz JM. Submental suction-assisted lipectomy without platysmaplasty: pushing the (skin) envelope to avoid a face lift for unsuitable candidates. Plast Reconstr Surg. 2003;112(5):1393-405.

18. Pitanguy I. The round-lifting technique. Facial Plast Surg. 2000;16(3):255-67.

19. Castro CC. The anatomy of the platysma muscle. Plast Reconstr Surg. 1980;66(5):680-3.

20. Castro CC. Superficial musculoaponeurotic system-platysma: a continuous study. Ann Plast Surg. 1991;26(3):203-11.

21. Daher M. Lipofacelift: plástica facial com descolamento mínimo da pele. Rev Bras Cir Plást. 2009;24(4):479-87.

22. Connell BF. Cervical lifts: the value of platysma muscle flaps. Ann Plast Surg. 1978;1(1):32-43.

23. Marchac D, Brady JA, Chiou P. Face lifts with hidden scars: the vertical U incision. Plast Reconstr Surg. 2002;109(7):2539-51.

24. Gomes Filho BS, Fleishmann Jr HW, Caldellas AV, Colombo FG, Andrade AAM. Ritidoplastia com cicatriz periauricular. Rev Bras Cir Plást. 2009;24(4):488-96.

25. Castro CC. The value of anatomical study of the platysma muscle in cervical lifting. Aesthetic Plast Surg. 1984;8(1):7-11.

26. Neves RE, Vasconcelos ZAA, Vasconcelos JJA. Terço médio da ritidoplastia cervicofacial. In: Mélega JM, Baroudi R, eds. Cirurgia plástica fundamentos e arte: cirurgia estética. Rio de Janeiro:Medsi;2005. p.25-42.

27. Aston SJ. Platysma muscle in rhytidoplasty. Ann Plast Surg. 1979;3(6):529-39.

28. Ellenbogen R, Karling JV. Visual criteria for success in restoring the youthful neck. Plast Reconstr Surg. 1980;66(6):826-37.

29. Castro CC, Aboudib JHC, Giaquinto MGC, Moreira MBL. Avaliação sobre resultados tardios em ritidoplastia. Rev Soc Bras Cir Plást. 2005;20(2):124-6.

30. Chia CY. Reposicionamento do terço médio na cirurgia da face: uma técnica simples de suspensão e de fixação. Rev Bras Cir Plást. 2008;23(2):71-4.

\section{Correspondência para:}

Daniel Nunes

Rua da Paz 129 apto 1 - Jardim dos Estados - Campo Grande, MS, Brasil - CEP 79002-190

E-mail: viva@dermatoeplastica.com.br 